\title{
Graphical Simulation for Design of a Tele-operated Clinker Clearing Robot
}

\author{
Jongwon Seo ${ }^{a^{*}}$, Graduate Research Assistant \\ Carl Haas ${ }^{a}$, Associate Professor \\ Alfred Traver ${ }^{b}$, Senior Lecturer \\ S.V. Sreenivasan ${ }^{b}$, Assistant Professor \\ Robert Stone ${ }^{\mathrm{b}}$, Graduate Research Assistant \\ Richard Greer ${ }^{b}$, Research Scientist \\ Chris Wunz ${ }^{\mathrm{a}}$, Graduate Research Assistant ${ }^{*}$ \\ ${ }^{a}$ Department of Civil Engineering \\ ${ }^{b}$ Department of Mechanical Engineering \\ University of Texas at Austin \\ Austin, TX 78712, USA
}

\begin{abstract}
Clinkers are waste material in the bottom ash hopper of furnaces of lignite fired power plants. Workers are required to wear cumbersome, hot suits and manipulate a long heavy steel rod connected to an air hammer to clear clinkers. A tele-operated maintenance robot has been designed to perform the dangerous clinker clearing operation. This paper describes three dimensional modeling and graphical simulation for design of the tele-operated clinker clearing robot. Graphical simulation provided valuable analysis at the design stage of the robot. The robot has to be operated in a constrained work environment, and the design of the robot has been improved and verified by analyzing interference and workspace with three dimensional CAD models.
\end{abstract}

\section{Introduction}

Maintenance and rehabilitation of existing power facilities can be dangerous and costly. Threats to health and safety are caused by an environment of tremendous potential energy sources, enclosed spaces, and hazardous working conditions. In the case of lignite fired power plants, clinkers which are waste material formed from the impurities in the lignite must be cleared from the bottom ash hopper when they block or plug the flushing apparatus. Clearing clinkers is a dangerous operation. Workers are required to wear cumbersome, hot suits and manipulate a long heavy steel rod connected to a jack hammer. Controliing the jack hammer and steel rod combination with their hands while in heavy protection suits exposes the workers to several safety hazards. The vibrations from the jack hammer can cause severe fatigue and internal damage to the workers. The hot and humid environment easily induces worker fatigue. Also, the end of the rod can swing upward and impact a worker if hit by a falling clinker. Clearing while the furnace remains in operation, even at reduced output, increases the danger substantially. Many similarities exist between construction work and the clinker clearing operation. The conventional method is laborious, physically dangerous, unpleasant and dirty, and requires large forces.

A tele-operated clinker clearing robot is being developed by the University of Texas at Austin for Houston Lighting and Power(HL\&P) and Electric Power Research Institute(EPRI). The developed robot will be operated in the HL\&P's Limestone

${ }^{\mathrm{a}^{*}}$ Correspond to jseo@mail.utexas.edu 
plant. This paper describes three dimensional modeling and graphical simulation for design of the tele-operated clinker clearing robot. Graphical simulation provides valuable analysis at the design stage of a robot. The robot has to work in constrained space, and many possible interferences with the existing structure exist. The robot design could be improved and verified through three dimensional graphical simulation. A case study of the use of graphical simulation for robot design is presented in this paper. The detailed description of the mechanical aspect of the robot is not included in this paper. The geometric aspect of the robot design is discussed.

\section{Geometric modeling of the work environment and the robot}

Three dimensional "as-built" solid models of the work environment and the robot were prepared with AutoCAD ${ }^{\mathrm{TM}}$ for graphical simulation. The furnace hopper structure and the outer environment which could affect the robot manipulation were modeled. Simplifications were made to help the modeling process and the visualization without losing critical information for analysis. Figure 1 is an isometric view of the work environment model. Virtual ceilings and walls which are not included in the figure were used to represent complex pipelines and the other outer structures. Once the work environment model was completed, the various ideas of the robot design could be experimented within the graphical work environment. The geometric model of the robot has been implemented from the conceptual design stage. After the first model was implemented, the design has been updated and improved by analyzing the graphical models. Basically, the robot has three main components. These are, an arm with a pneumatic hammer attached at the end, an actuator frame with arm rotation and insertion mechanisms, and a mating frame. Figure 2 shows the simplified model of the robot.

\section{Operating sequence and interference analysis}

The robot is designed to be attached to the hopper structure so that it can perform the clearing operation through the access door of the hopper. The hopper is filled with water and it needs to be flushed before the clearing operation. During flushing, the actuator frame which includes critical components should be kept out of the way of the flushed hot water. Therefore, it is designed to swing open. After flushing, the actuator frame is positioned to its working location. Figure 3 shows the configuration of the robot when the actuator frame is open and closed. The robot arm is inserted into the insertion mechanism after the actuator frame is closed and settled to its working position. The robot starts its operation after this installation process. The installation process and the operation involves possible interference between the robot and the work environment. Any modification of the existing structure was not desirable, so the interferences were carefully checked for the geometric design of robot. The interference between the robot arm and the existing structure during the clearing operation is discussed in the workspace analysis section. The following interferences were avoided through graphical simulation:

- Access Door and Actuator Frame - Actuator Frame in Working Position

After the flushing operation, the actuator frame gets into the working position. At that time, the access door is open, and the collision between the actuator frame and the door should be avoided. The right side of the actuator frame has been designed to accommodate the door safely. Figure $3(\mathrm{~b})$ shows the access door accommodated by the actuator frame.

- Camera Enclosure and the Opening

The CCTV camera for visual feedback is protected with the camera enclosure from the heat of the bottom hopper. It is designed to rotate with the robot arm. However, it could cause collisions with the access opening and the actuator frame. After the enclosure movement was specified, the proper outside shape and size of the enclosure was selected. Figure 4 shows the collision-free camera movement.

\section{- Insertion Mechanism and Actuator Frame} The robot arm is a straight steel tube and the orientation of the arm is controlled by rotating the insertion mechanism about a gimbal. This operation involves the interference between the insertion mechanism and the actuator frame. The limitation of the rotation of the insertion mechanism was identified with the interference analysis. Figure 5 shows the collision-free movement of the insertion mechanism.

\section{Workspace analysis}


Basically, the arm has three degrees of freedom which are vertical and horizontal rotation, and a prismatic motion for insertion and retraction. These degrees of freedom are driven with hydraulically actuated cylinders. After the pneumatic hammer attached at the end of the arm is positioned to clinkers, the hammer is activated and breaks clinkers so that they can be handled with the grinder. It was important to analyze the workspace of the arm and to determine the proper length of the arm. The inner and outer interferences limit the workspace of the robot, so the workspace should be determined considering the interferences. The robot arm is not telescopic. The whole arm has to move back and forth for retraction and insertion. It is designed that way because an expensive telescopic arm can be damaged when clinkers can fall onto the arm. The arm should be long enough to be able to reach far away from the access door. However, a long arm cannot operate in the area closer to the access door because of interference with outer structures. It was decided to use two types of arms(long and short). Each arm is designed to compensate for each other's workspace limitation.

The work space analysis has been done with an AUTOLISP routine. AUTOLISP is an interface language for AutoCAD ${ }^{\mathrm{TM}}$. The routine iterates interference checking between the arm and the existing structures by changing the insertion length and the rotation angles of the arm in small amounts. The result of the routine is a representation of the reachable volume with a given length of the robot arm. An example of the result of the workspace analysis is presented in Figure 6. The figure shows the top view of the furnace hopper structure, and the horizontally reachable area of two arms at a fixed vertical rotation configuration was represented. The gray-colored area shows the reachable area with the short arm which was not accessible with the long arm. The work space can be visually verified by running the routine with the variation in vertical rotation angles. AutoCad is just a static $\mathrm{CAD}$ software. However, the dynamic simulation for thorough workspace and interference analysis was possible with the interface language.

The robot configuration which produces collisions can be identified with the routine. Realtime collision avoidance will be achieved by making a look-up table for the collision-free configuration of the robot. The look-up table will be stored in the control computer memory and the operator will be prohibited from the collision.

\section{Communication between the robot designers and the field workers through animation}

The robot will replace the workers in hazardous areas. The communication between robot designer and the field workers who have been doing the clearing operation manually was critical to check the design of the robot. The robot design and its operation has been animated for better communication. A animation software 3D Studio ${ }^{\mathrm{TM}}$ has been used to animate the static CAD models. 3D Studio $^{\mathrm{TM}}$ is not a robot simulation program, but the robot installation process and the operation could be clearly animated with rendered images. It helped the design process with the feedback from field workers.

\section{Future plan for graphical control}

Currently, the robot is designed to be remotely controlled with the visual feedback from CCTV. It is anticipated that the visual feedback from CCTV may be limited for the tele-operation if the ash obscures the vision. The robot will be graphically controlled in the future to overcome the limitation of the visual feedback. The three dimensional graphical models of the work environment and the robot will be used to control the actual robot. The models will be updated with various sensors. The robot configuration can be updated with encoders of the hydraulic cylinders, and the environmental sensors will be needed to update the location and the shape of clinkers. By graphically driving the robot, the operator can simulate different views and zooms with the graphical environment, and better spatial perception and peripheral views can be provided to the operator. The robots movement can be graphically simulated and verified for safe operation before actual execution. It can also be used for operator training.

\section{Conclusion}

A tele-operated clinker clearing robot has been designed through three dimensional geometric modeling and graphical simulation. Graphical simulation improved the design of the robot. The robot behaviors can be simulated within the graphical work environment, and every step of the operating sequence can be verified with graphic models. Results of interference analysis can also be used for real time control. 


\section{Acknowledgment}

The authors wish to thank HL\&P and EPRI for their support for this research.

\section{References}

1) Carter, H.R., Koksal, C.G., and Garrabrant, M.A., "Furnace Cleaning in Utility Boilers Burning Powder River Basin Coals," American Society of Mechanical
Engineers, 92-JPGC-Pwr-35, pp.1-7, NewYork, ASME.

2) Haas, C.T., "Feasibility Study of Alternative Clinker Clearing Solutions," Dept. of Civil Engineering, University of Texas, TX, 1995.

3) Haas, C.T., et. Al., "Development of Tele-operated Robotic Maintenance Equipment and Dynamic Graphical Interface", Annual Progress Report for Electric Power Research Institute and Houston Lighting and Power, 1996.

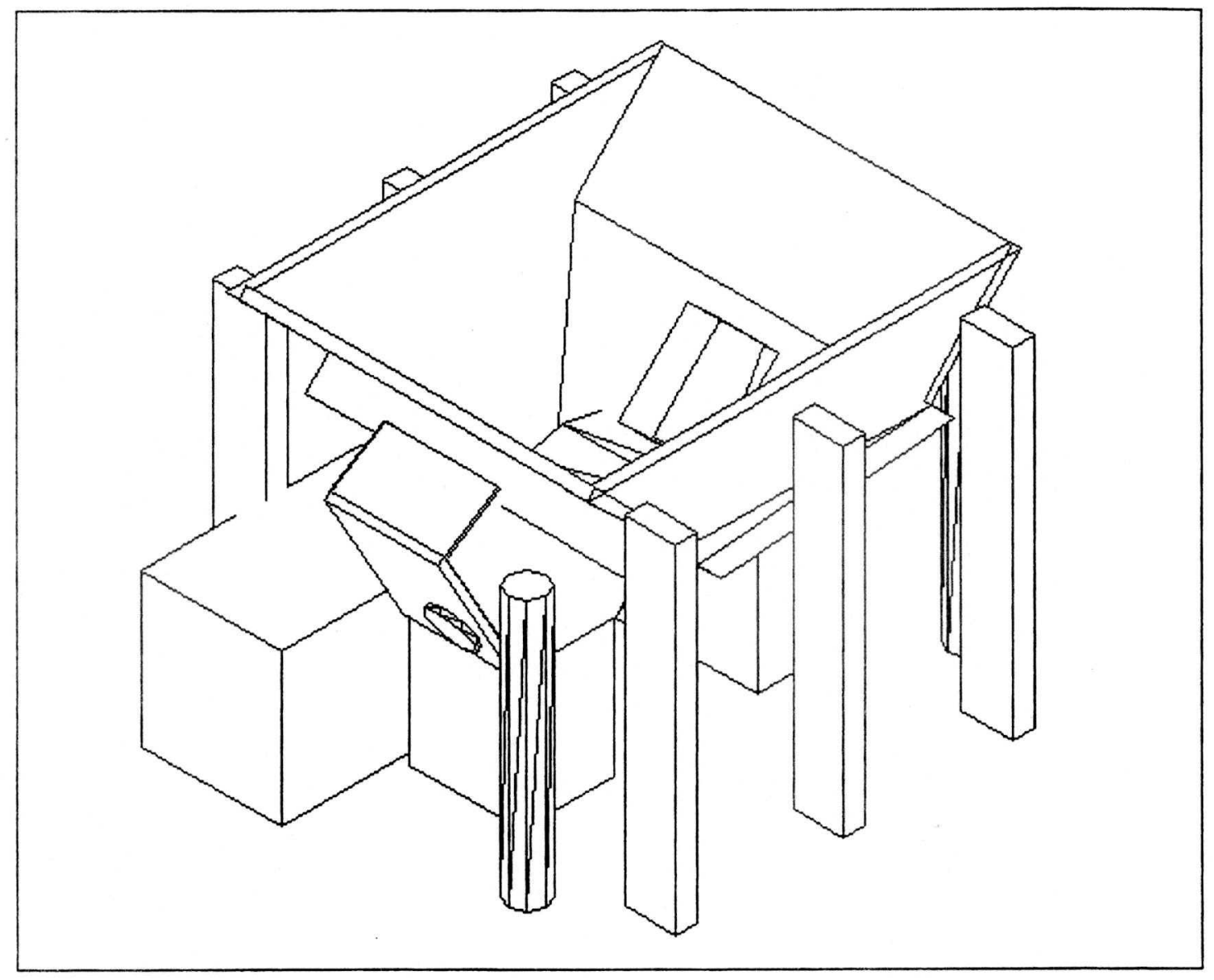

Figure 1. Work Environment Model 


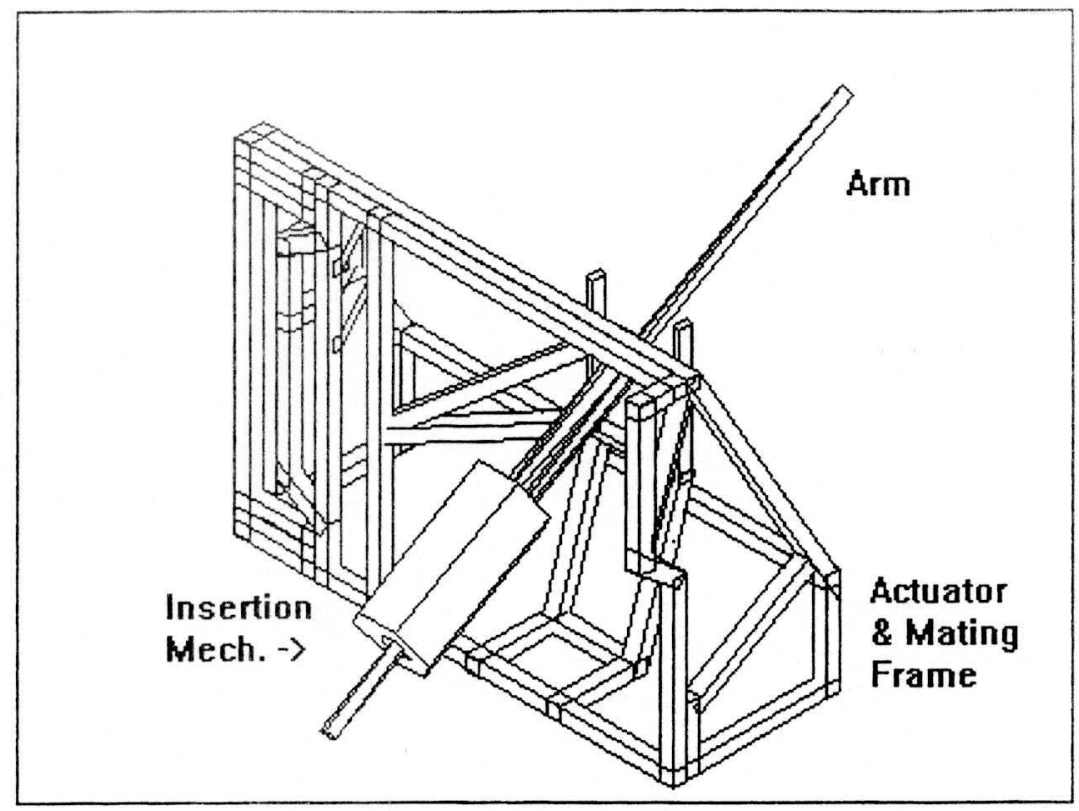

Figure 2. Robot Model

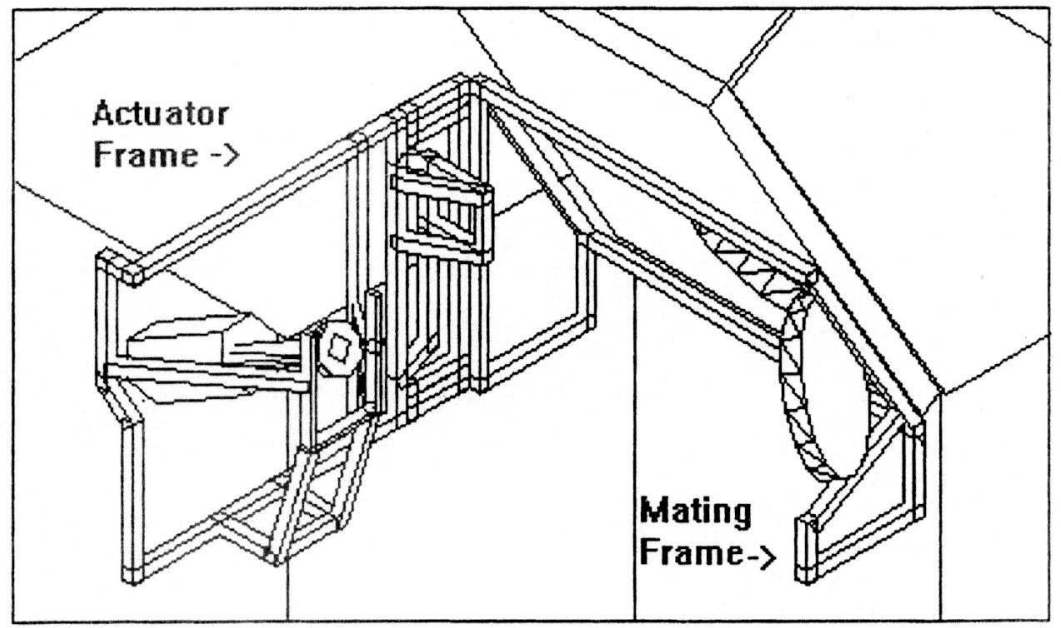

Figure 3. a) Actuator Frame Open

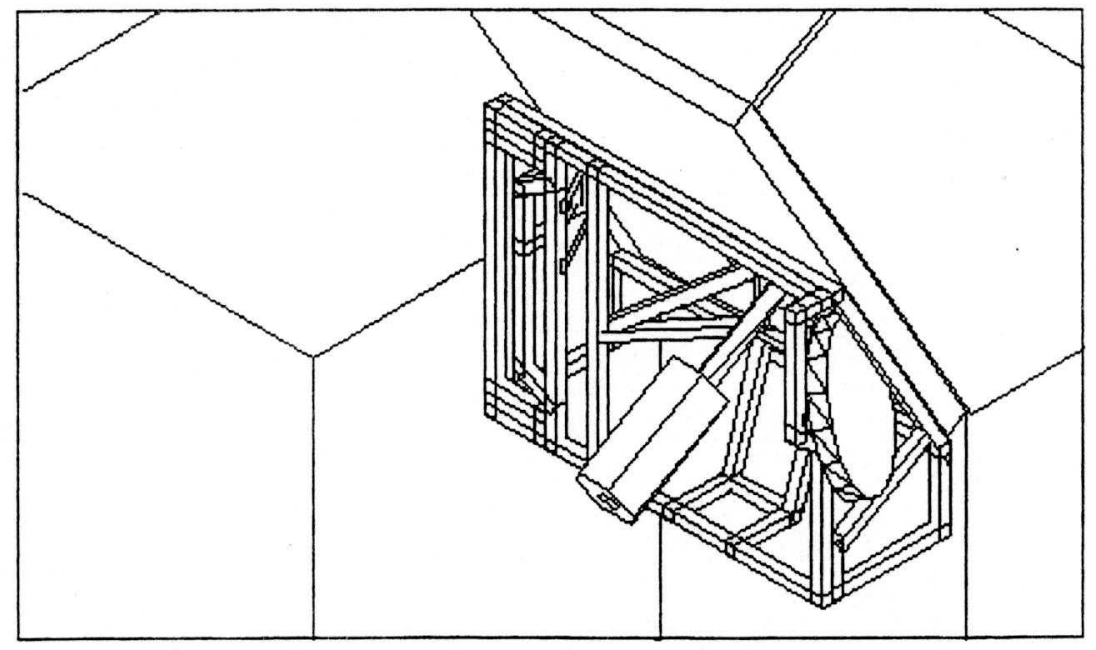

Figure 3. b) Actuator Frame Closed(Working Position) 


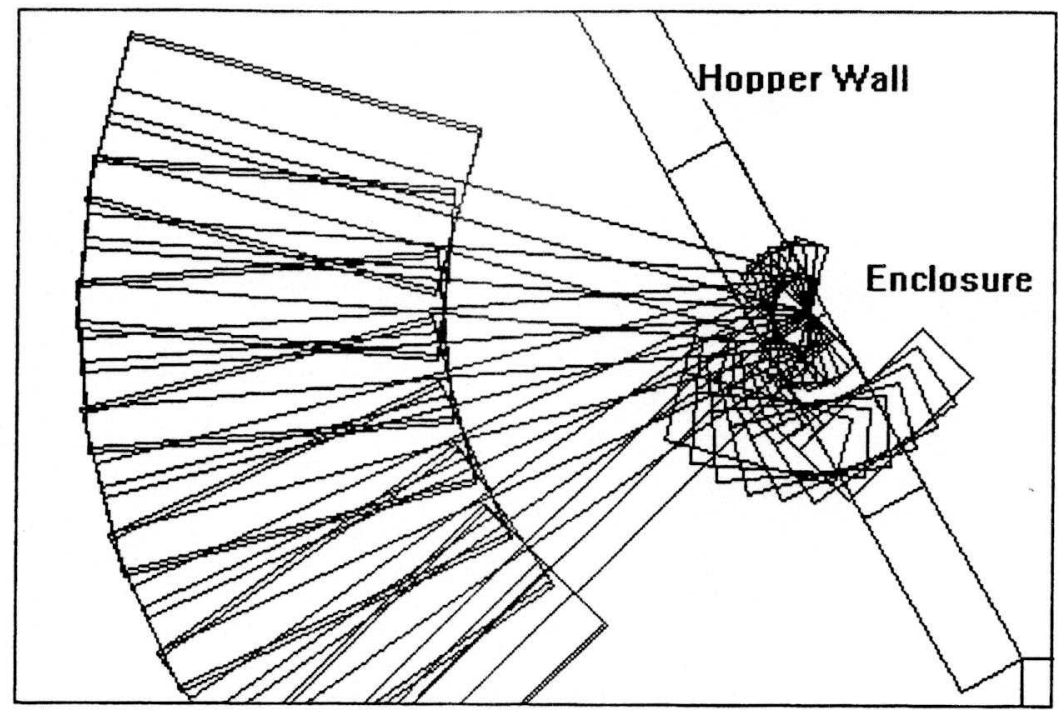

Figure 4. Collision-Free Camera Movement

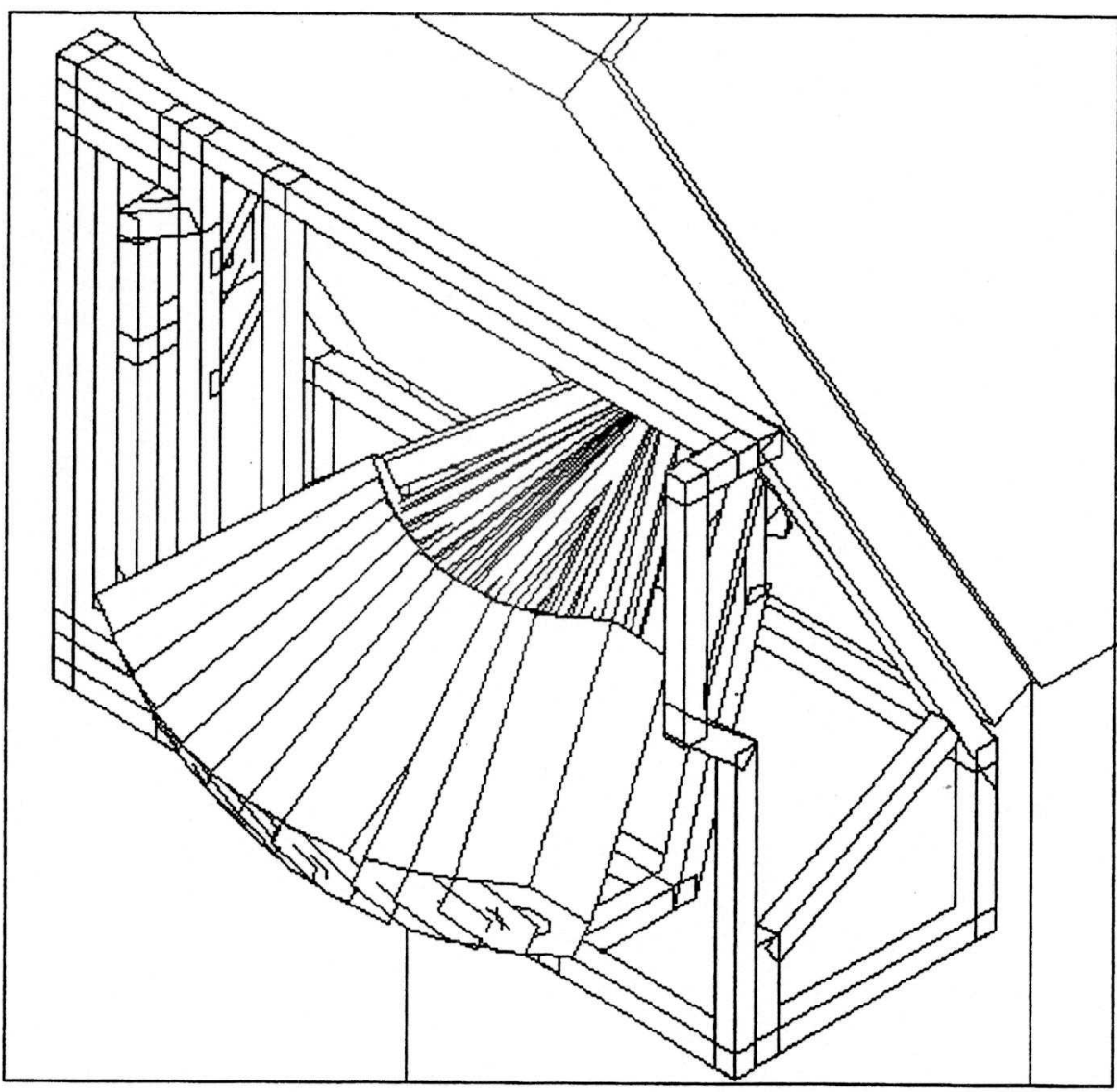

Figure 5. Collision-Free Insertion Mechanism Movement 


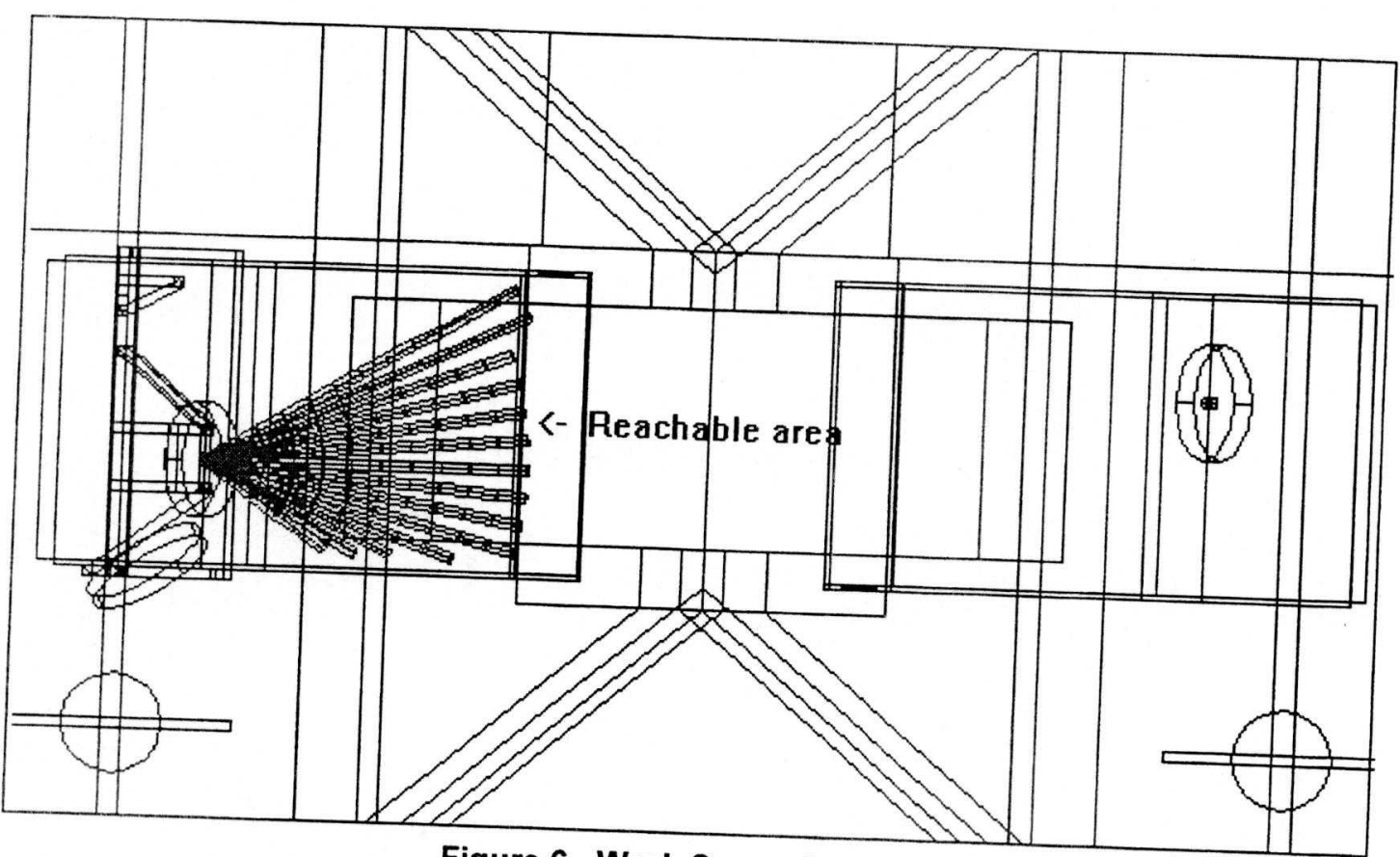

Figure 6. Work Space Analysis 